\title{
Perception de la chimioprévention du paludisme saisonnier au Niger
}

\author{
Issa SALISSOU ${ }^{1,2}$, Lamine MAHAMAN MOUSTAPHA ${ }^{3}$, Bako YERIMA ${ }^{3}$, \\ Ibrahim ALKASSOUM ${ }^{4}$, Djakou HADIZA ${ }^{5}$ et Maman Laminou IBRAHIM ${ }^{3 *}$ \\ ${ }^{I}$ Institut de Santé Publique de Niamey, Niger. \\ ${ }^{2}$ Hôpital de District de Magaria, Niger. \\ ${ }^{3}$ Centre de Recherche Médicale et Sanitaire de Niamey, Niger. \\ ${ }^{4}$ Direction de la Surveillance et de la Riposte aux Epidémies, Niamey, Niger. \\ ${ }^{5}$ Programme National de Lutte contre le Paludisme. Niamey, Niger. \\ *Auteur correspondant ; E-mail : lamine@cermes.org; 634 Bd de la Nation, YN 034 Niamey-Niger. \\ BP : 10.887. Tel +227207520 40. Fax : +22720753180.
}

\section{RESUME}

La chimioprévention du paludisme saisonnier (CSP) est une nouvelle stratégie recommandée par l'Organisation Mondiale de la Santé (OMS) depuis mars 2012 aux pays où la transmission du paludisme est saisonnière. Le programme national de lutte contre le paludisme (PNLP) du Niger a initié une étude pilote en 2013 au niveau du district sanitaire de Magaria avec l'appui de Médecins Sans Frontières (MSF) avant sa mise à l'échelle nationale. C'est dans ce contexte qu'une enquête a été menée pour évaluer la perception de la CPS par la population à Magaria. Quatre-vingt-quatre pourcents des parents sont satisfaits de la chimioprévention du paludisme saisonnier. Le taux de couverture de la CPS (4 passages) est de $81,3 \%$. Quatre-vingt-neuf virgule deux pourcents ont respecté l'observance du traitement. Aucun évènement indésirable majeur n'a été enregistré. Cependant, $27.8 \%$ des enfants ont manifesté des évènements indésirables mineurs. Les principales manifestations sont la diarrhée $(53,7 \%)$ et le vomissement (38,8\%). La CPS est une stratégie acceptée par la population. Le taux de couverture et l'observance du traitement sont très élevés et les effets secondaires sont mineurs. Au vu de ces résultats, le programme national de lutte contre le paludisme peut mettre en œuvre la CPS à l'échelle nationale.

(C) 2016 International Formulae Group. All rights reserved.

Mots clés : Enquête, perception, chimioprévention du paludisme saisonnier, Niger.

\section{Perception of the seasonal malaria chemoprevention in Niger}

\begin{abstract}
Seasonal Malaria Chemoprevention (SMC) is a new strategy recommended by the World Health Organization since March 2012 for countries with seasonal malaria transmission. The National Malaria Control Programme (NMCP) of Niger has initiated a pilot study in the health district of Magaria in children of 3-59 months, with the support of Médecins Sans Frontières. In this context, we have conducted a questionnaire to assess how the population perceives the implementation of SMC. By 241 parents, $84 \%$ of parents are satisfied with seasonal malaria chemoprevention. SMC coverage (4 cycles) was $81.3 \%$ and $89.2 \%$ complied with treatment adherence. No severe adverse effect was recorded. However, $27.8 \%$ of the children showed minor
\end{abstract}


adverse effects. The main adverse effects reported were diarrhea (53.7\%) and vomiting (38.8\%). SMC is a strategy well accepted by the population and the coverage and treatment compliance are very high. The adverse effects are also minor. SMC could be implemented at a larger scale in Niger by the NMCP.

(C) 2016 International Formulae Group. All rights reserved.

Keywords: Perception, seasonal malaria chemoprevention, Niger.

\section{INTRODUCTION}

Depuis mars 2012, l'OMS a recommandé une nouvelle stratégie appelée CPS pour prévenir le pic de morbimortalité chez les enfants de moins de cinq ans des pays de la région du Sahel (OMS, 2014). Le Niger est un pays sahélien soumis à d'importants changements climatiques (Habou et al., 2016). La saison des pluies s'étend de mi-juin à octobre qui coïncide avec la période de transmission du paludisme (Morou et al., 2016). La CPS consiste à l'administration intermittente d'un traitement complet de Sulfadoxine Pyriméthamine + l'Amodiaquine pendant la saison de haute transmission. L'objectif est de maintenir des concentrations thérapeutiques de ces médicaments dans le sang pendant la période où le risque de contracter le paludisme est plus élevé (Carneiro et al., 2010). Cette stratégie a été mise en œuvre au niveau de cinq districts sanitaires pilotes: Guidan Roumdji, Bouza, Madaroufa, Madaoua et Magaria en collaboration avec des ONG comme Médecins Sans Frontière (MSF). C'est dans ce contexte, qu'une enquête a été conduite pour évaluer la perception de la CPS par la population au niveau de six districts sanitaires de Magaria. Il s'agit de façon spécifique de déterminer leur connaissance, leurs pratiques et leurs aptitudes, calculer la couverture de CPS, déterminer l'observance du traitement et enfin déterminer la proportion des évènements indésirables et leur nature.

\section{MATERIEL ET METHODES Type d'étude}

Il s'agit d'une enquête transversale pour évaluer la perception de la chimioprévention du paludisme saisonnier par la population de Magaria. L'enquête a été conduite du 23 mars 2014 au 23 juin 2014.

\section{Site d'étude}

Cette étude est conduite au niveau de six Centres de Santé Intégré (CSI) (Bangaza, Dantchiao, Dogo Dogo, Dungas, Magaria et Mallaoua) du district sanitaire de Magaria. Ce dernier est situé dans la partie Sud de la région de Zinder (Niger). Il couvre une superficie de $7.903 \mathrm{Km}^{2}$. Le climat est de type sahélo soudanien caractérisé par une saison sèche (Octobre à mai) et une saison pluvieuse (Juin à Septembre). La pluviométrie varie de 500 $\mathrm{mm}$ à $700 \mathrm{~mm}$ par an.

\section{Population d'étude}

La population d'étude est constituée par les parents des enfants de 3 à 59 mois. En 2013, la population de Magaria est estimée à 744268 habitants avec une densité de 94 habitants $/ \mathrm{km}^{2}$. Elle est composée de quatre groupes ethniques: Haoussa, Peuls, Kanouri et Touaregs.

\section{Echantillonnage et collecte des données}

Une quarantaine de ménages a été enquêtée dans chacun des six CSI. Cette taille est calculée sur la base qu'aucune étude sur la CPS n'a été faite. Nous avons pris une prévalence de $50 \%$ et une précision de $10 \%$ pour calculer la taille de l'échantillon. $\mathrm{N}=\mathrm{Z} \alpha^{2}$ $(1-p)^{*} p / d^{2} . \quad n=1,96^{2} \quad 0,50(1-0,5) / 0,10^{2}=96$. Avec: $\mathrm{n}=$ la taille de l'échantillon. $\mathrm{d}=$ précision qui est de $10 \%, Z \alpha=1,96$ avec une marge d'erreur $\alpha=5 \%, p=$ proportion $d u$ phénomène dans la population $=0,5$. La taille finale est de 240 mères d'enfants en raison de 40 enfants par CSI. La collecte des données a été faite à l'aide de questionnaire aux mères des enfants de 3 à 59 mois. Les outils de collecte sont constitués par des questionnaires. 


\section{Ethique}

Le projet a préalablement été présenté au comité national consultatif d'éthique qui l'a approuvé. Un consentement éclairé a été obtenu des participants de l'étude.

\section{Analyse des données}

Toutes les données des fiches d'enquêtes ont été saisies dans une feuille Excel du logiciel Microsoft Office. Les données ont été analysées grâce au logiciel Epi Info 3.2.10.

\section{RESULTATS}

\section{Caractéristiques de la population d'étude}

Deux cents quarante-un parents d'enfants au niveau de six CSI où la CPS est mise en œuvre ont été questionnés. L'âge moyen des enfants était de 2,2 ans $(\alpha=1,32$; $[0,24,5])$ et le sexe ratio de 1,06 en faveur des filles.

\section{Connaissances, aptitudes et pratique de la population}

Quatre-vingt-sept virgule neuf pourcents des parents connaissaient la chimioprévention du paludisme saisonnier. Les agents de santé communautaire constituent le moyen de communication le plus important et représentent $83,4 \%$. Seuls $6,64 \%$ des parents d'enfants ont un niveau secondaire d'instruction, $18,26 \%$ ont un niveau primaire d'instruction et $75,1 \%$ n'ont aucune instruction (Figure 1).

\section{Satisfaction des parents de la CPS et observance du traitement \\ Quatre-vingt-quatre virgule deux} pourcents des parents affirment être satisfaits de la CPS car elle réduirait les épisodes du paludisme et les fréquentations des centres de santé. Quatre-vingt-neuf virgule deux pourcents des parents ont respecté l'observance au traitement.

\section{Couverture de la CPS et évènements indésirables}

Le taux de couverture (4 passages) de la CPS était de $81,33 \%$. Toutefois, la couverture est très variable d'un CSI à un autre. Ce taux de couverture est de $100 \%$ à Bangaza, $82,5 \%$ à Dantchiao, $45 \%$ à Dogo Dogo, $75 \%$ à Dungass, $97,5 \%$ à Magaria et $90 \%$ à Mallaoua (Figure 2). Vingt-sept virgule huit pourcents des parents d'enfants affirment que leurs enfants ont eu des évènements indésirables suite à la prise des médicaments de la CPS. Aucun évènement indésirable majeur de type syndrome de Lyell et Steven Janson n'a été enregistré. Par contre des évènements indésirables mineurs ont été observés. Le principal évènement indésirable est l'intolérance au traitement qui se traduit par de la diarrhée $(53,73 \%)$ ou des vomissements $(38,8 \%)$.

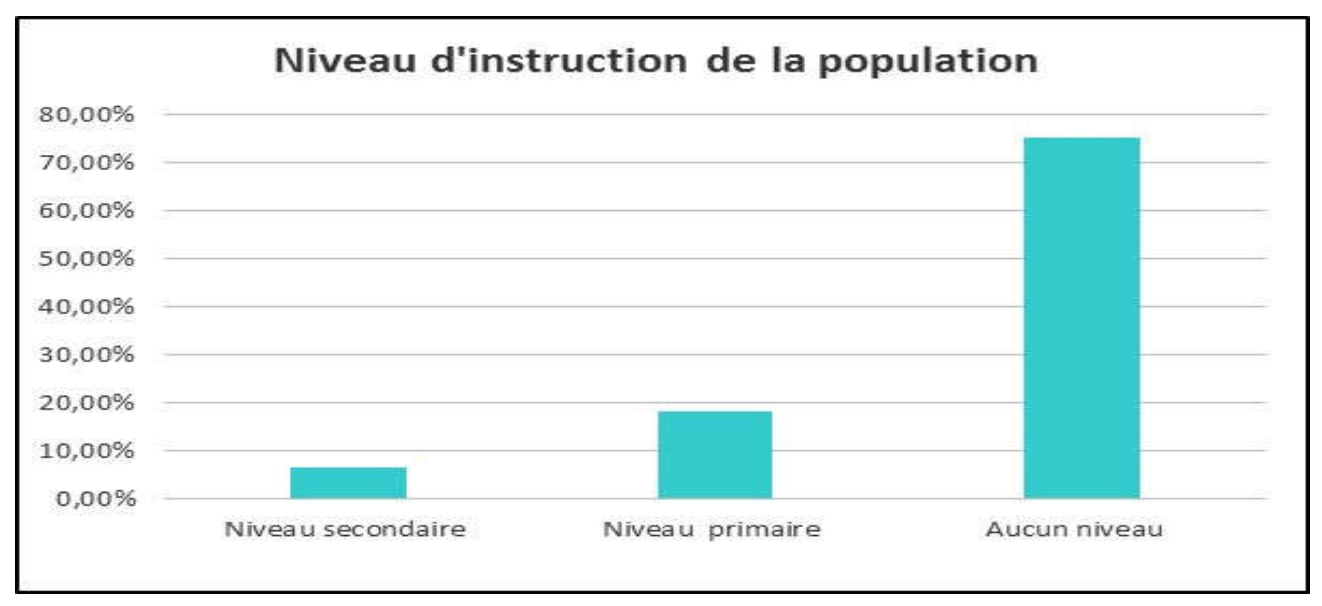

Figure 1 : Niveau d'instruction de la population à Magaria. 


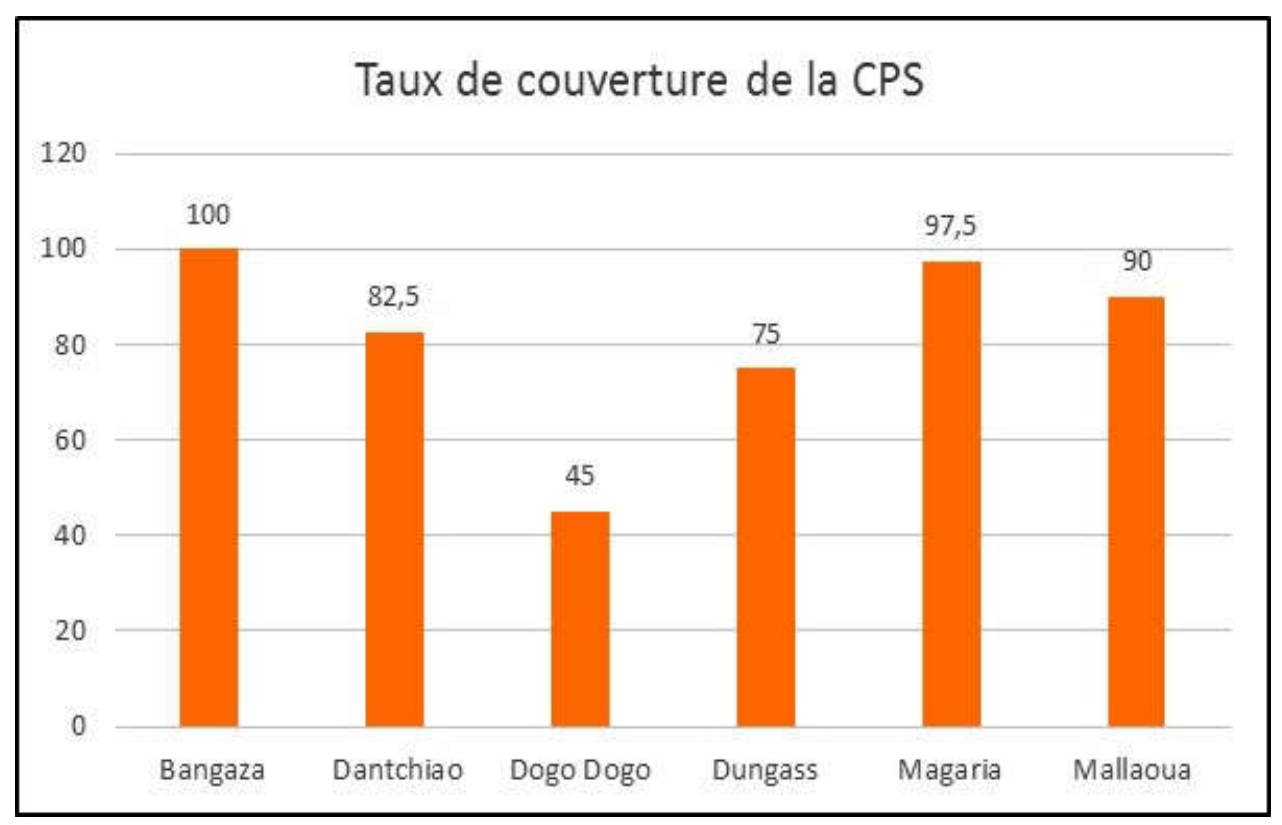

Figure 2: Taux de couverture la CPS à Magaria.

\section{DISCUSSION}

Cette étude est la première évaluation de la perception de la chimioprévention du paludisme saisonnier, après celle ayant montré l'éligibilité du Niger pour cette nouvelle stratégie (Guillebaud et al., 2013). Elle permettra sans nul doute au programme national de lutte contre le paludisme de mettre à l'échelle nationale cette stratégie.

Quatre-vingt-quatre virgule deux pourcents des parents sont satisfaits de la chimioprévention du paludisme saisonnier. Ce qui explique l'observance des traitements et l'excellent taux de couverture (proportion d'enfant ayant reçu le traitement complet pendant les 4 passages). En effet, 89,2\% des parents ont bien observé le traitement et la couverture de la CPS est de $81.3 \%$. Ce taux de couverture est variable selon le CSI avec des valeurs extrêmes à Bangaza (100\%) et Dogo Dogo (45\%). Quatre-vingt-sept virgule quatrevingt-dix-sept pourcents de la population est informée de la campagne de lancement de la CPS et le principal canal de transmission de l'information est représenté par les agents de santé communautaire $(83,4 \%)$. Cette satisfaction s'explique aussi par la réduction significative de la morbidité attribuable au paludisme. Toutefois, le niveau d'instruction de la population est très faible. En effet, $75,1 \%$ de la population n'est pas instruite. Ce qui rend difficile la formation et la mise œuvre de la CPS. Cependant, comme au Sénégal, on peut combiner la CPS à la prise en charge du paludisme à domicile qui est faite par les agents de santé communautaire mieux instruits (Tine et al., 2013; Tine et al., 2014). Parmi les causes de refus de la CPS, il y'a les évènements indésirables dont la proportion est de $27,8 \%$. Le principal évènement indésirable est l'intolérance au traitement qui se traduit par la diarrhée ou le vomissement. En effet, l'amodiaquine a un goût très amère d'où le nombre élevé de rejets après son absorption. Aussi, parmi les causes de refus de la CPS il y'a l'absence de maladie. En effet, certains parents refusent la CPS parce que leur enfant n'est pas malade. La CSP est une stratégie préventive et non curative. On doit insister sur cet aspect 
préventif lors des formations des futures campagnes CPS. Ce point ne fut pas inscrit dans le questionnaire et constitue la principale limite de cette étude. La CPS sera bientôt une stratégie supplémentaire à la lutte intégrée contre le paludisme en Afrique au Sud du Sahara, au même titre que le contrôle des vecteurs par les insecticides (Ahadji-Dabla et al., 2014), la prise en charge des malades par les combinaisons thérapeutiques à base d'artémisinine (Kpaibe et al., 2016), la prévention du paludisme chez la femme enceinte par le traitement préventif intermittent et même la médecine traditionnelle par divers plantes en milieu rural (Denou et al., 2016).

\section{Conclusion}

La chimioprévention du paludisme saisonnier est une stratégie acceptée par la population du Niger. Le taux de couverture et l'observance du traitement sont très élevés et justifient cette acceptation. Les évènements indésirables au traitement sont mineurs. Le PNLP peut mettre en œuvre à l'échelle nationale cette nouvelle stratégie au Niger.

\section{CONFLIT D'INTERETS}

Les auteurs déclarent qu'il n y'a pas de conflit d'intérêts.

\section{CONTRIBUTIONS DES AUTEURS}

IML a conçu le projet et rédigé l'article; MML a participé la rédaction de l'article; SI et YB ont mis en œuvre du projet; HJ a aidé à trouver le financement ; AI a participé à la conception du projet et à la rédaction de l'article.

\section{REMERCIEMENTS}

Nous remercions Médecin Sans Frontière Suisse pour avoir mis en ouvre la chimioprévention du paludisme dans le district de Magaria. Nous remercions Yerima Bako, médecin chef de l'hôpital du District Sanitaire d'avoir permis la réalisation de cette étude dans son district. Nous remercions enfin l'Institut de Santé Publique qui a inscrit ce projet dans la liste des mémoires du diplôme d'étude spécialisée pour l'année 2013-2014.

\section{REFERENCES}

Ahadji-Dabla KM, Ketoh GK, Nyamador WS, Apetogbo GY, Glitho IA. 2014. Susceptibility to DDT and Pyrethroids, and Detection of Knockdown Resistance Mutation in Anopheles Gambiae Sensu Lato in Southern Togo. International Journal of Biological and Chemical Sciences, $\quad \mathbf{8}(1): \quad 314-23$. Doi:10.4314/ijbcs.v8i1.27.

Carneiro I, Smith L, Ross A, Roca-Feltrer A, Greenwood B, Armstrong Schellenberg J, Smith T, Schellenberg D. 2010. Intermittent Preventive Treatment for Malaria in Infants: A Decision-Support Tool for Sub-Saharan Africa. Bulletin of the World Health Organization, 88 (11): 807-14. Doi:10.2471/BLT.09.072397.

Denou A, Koudouvo K, Haidara M, Togola A, Sanogo R, Essien K, Aklikokou KA, Diallo D, Gbeassor M. 2016. Activité Analgésique de Quatre Plantes Utilisées Dans La Prise En Charge Traditionnelle $\mathrm{Du}$ Paludisme $\mathrm{Au}$ Mali et $\mathrm{Au}$ Togo. International Journal of Biological and Chemical Sciences, 10(3): 1342-49. Doi.10.4314/ijbcs.v10i3.34

Guillebaud J, Mahamadou A, Zamanka H, Katzelma M, Arzika I, Maman LI, Elfatih E, et al. 2013. Epidemiology of Malaria in an Area of Seasonal Transmission in Niger and Implications for the Design of a Seasonal Malaria Chemoprevention Strategy. Malaria Journal 12(1): 379. Doi:10.1186/14752875-12-379.

Habou ZA, Mahamadou KB, Toudou A. 2016. Les Systèmes de Productions Agricoles $\mathrm{Du}$ Niger Face $\mathrm{Au}$ Changement Climatique: Défis et Perspectives. International Journal of Biological and Chemical Sciences, 10(3): 1262-72. Doi:10.4314/ijbcs.v10i3.28

Kpaibe AS, Bony FN, Khra Yoboue L, Komenan Gbassi G, N'cho Amin C, Ake M, Kla Malan A. 2016. Stabilité Des 
Suspensions Reconstituées de La Combinaison Thérapeutique Antipaludique À Base D'artémétherLuméfantrine Avec Différents Types D'eaux. International Journal of Biological and Chemical Sciences, 10(2): 894-903. Doi.10.4314/ijbcs.v10i2.36

Morou B, Ounani H, Oumani AA, Diouf A, Guero C, Mahamane A. 2016. Caractérisation de La Structure Démographique Des Ligneux Dans Les Parcs Agroforestiers Du Terroir de Dan Saga (Aguié, Niger). International Journal of Biological and Chemical Sciences, $\quad$ 10(3): $\quad 1295-1311$. Doi:10.4314/ijbcs.v10i3.31

Organisation Mondiale de la Santé. Chimioprévention $\mathrm{Du}$ Paludisme Saisonnier Par Administration de Sulfadoxine-Pyriméthamine et D'amodiaquine Aux Enfants: Guide de Terrain. 2014. WHO. Accessed October 29. http://www.who.int/malaria/publications/ atoz/9789241504737/fr/.

Tine RCK, Ndour CT, Faye B, Cairns M, Sylla K, Ndiaye M, Ndiaye JL, et al. 2014. Feasibility, Safety and Effectiveness of Combining Home Based Malaria Management and Seasonal Malaria Chemoprevention in Children Less than 10 Years in Senegal: A Cluster-Randomised Trial. Transactions of the Royal Society of Tropical Medicine and Hygiene, 108 (1): 13-21. Doi:10.1093/trstmh/trt103.

Tine RCK, Ndiaye P, Ndour CT, Faye B, Ndiaye JL, Sylla K, Ndiaye M, et al. 2013. Acceptability by Community Health Workers in Senegal of Combining Community Case Management of Malaria and Seasonal Malaria Chemoprevention. Malaria Journal 12(1): 467. Doi:10.1186/14752875-12-467. 